\title{
Increased antenatal HIV testing among rural pregnant women: a community-based HIV prevention services program in rural Maharashtra, India
}

Ashok Dyalchand*, Gita Sinha

From $16^{\text {th }}$ International Symposium on HIV and Emerging Infectious Diseases

Marseille, France. 24-26 March 2010

\section{Background}

To characterize changes in antenatal HIV testing utilization, six months after the introduction of a targeted HIV prevention and testing services intervention program.

\section{Methods}

Between August 2006 and April 2007, a communitybased HIV prevention campaign and antenatal clinicintegrated voluntary HIV testing program was initiated in 52 villages in rural Aurangabad district, Maharashtra, India. Questionnaires assessing HIV risk factors and HIV testing utilization were administered to random cross-sectional community samples of 400 adult women who had given birth in the prior 12 months, at Baseline and, after six months, to 400 women each in the PostIntervention and a separate Control community.

\section{Results}

During the study period, a total of 1200 recently-pregnant women were surveyed. Sociodemographic characteristics including $>90 \%$ antenatal care utilization were similar for all sample groups. From Baseline to PostIntervention, HIV testing facility awareness significantly increased $(6 \%$ to $16 \%, \mathrm{p}<0.05)$ and independently correlated only with participation in the community-based campaign (AOR 2.1, 95\% CI (1.3-3.5)). Antenatal HIV testing utilization increased $(3.3 \%$ vs $7.5 \%, \mathrm{p}<0.05)$, and correlated with discussing HIV in antenatal care (AOR $10.2,95 \%$ CI $(4.7-22.4)$ ) but did not vary with STI symptom history. Of Post-Intervention women reporting antenatal HIV testing ( $\mathrm{n}=30), 43 \%$ sought testing at known voluntary HIV testing facilities, including Intervention clinics and the District-level hospital.

\section{Discussion}

The community- and clinic-integrated HIV prevention services program significantly raised women's HIV testing awareness and the number of clinic-integrated facilities for voluntary HIV testing, influencing increased community-level antenatal HIV testing utilization. In all rural communities, it is necessary for current antenatal services to identify and counsel the high-HIV risk subgroup of women with STI symptom history, and to ensure voluntary informed consent, for all clients.

Published: 11 May 2010

\section{doi:10.1186/1742-4690-7-S1-P146}

Cite this article as: Dyalchand and Sinha: Increased antenatal HIV testing among rural pregnant women: a community-based HIV prevention services program in rural Maharashtra, India. Retrovirology 2010 7(Suppl 1):P146

* Correspondence: dyalchand@gmail.com

Institute of Health Management Pachod, Pune, India 